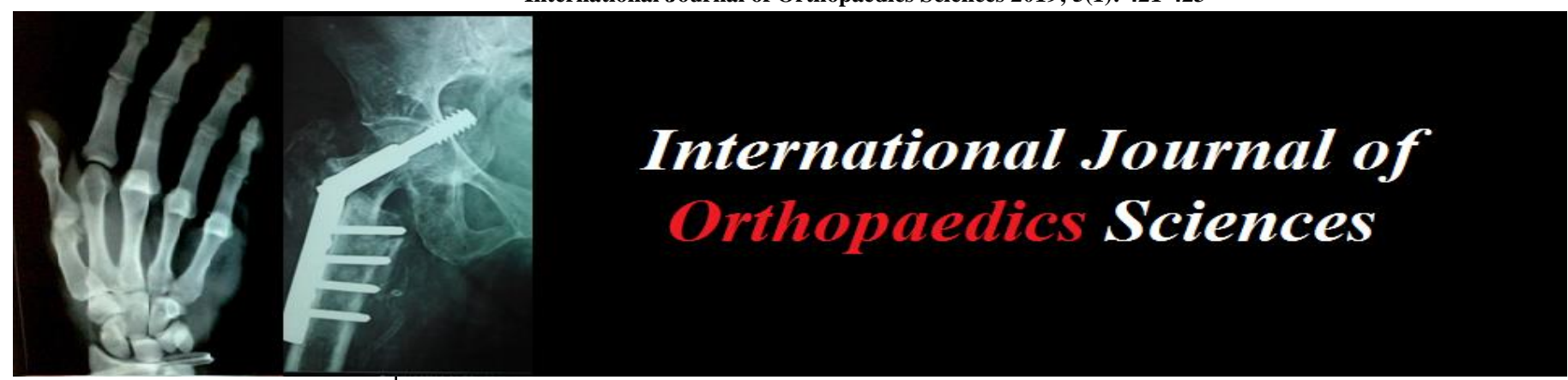

ISSN: 2395-1958

IJOS 2019; 5(1): 421-423

(C) 2019 IJOS

www.orthopaper.com

Received: 16-11-2018

Accepted: 19-12-2018

Dr. Saral Patel

M.S. Orthopedics, Assistant

Professor, B.J. Medical College,

Ahmedabad, Gujarat, India

Dr. Mitul Mistry

M.S. Orthopedics, Assistant

Professor, B.J. Medical College,

Ahmedabad, Gujarat, India
Correspondence

Dr. Mitul Mistry

M.S. Orthopedics, Assistant

Professor, B.J. Medical College,

Ahmedabad, Gujarat, India

\section{A prospective study of results of laminectomy using ultrasonic bone scalpel $v s$. conventional methods}

\section{Dr. Saral Patel and Dr. Mitul Mistry}

DOI: https://doi.org/10.22271/ortho.2019.v5.i1h.76

\section{Abstract}

Dural tear is the most unintended complication during spinal decompressive surgeries. In routine methods of spinal decompression like in congenital stenotic lesion, degenerative/spondylotic changes there is increased incidence of dural injuries due to thin dura and harder bone. High-speed drills and diamond burrs increases heat production and causes dural damage. Ultrasonic bone scalpel (UBS) in spinal laminectomy is gaining popularity due to numerous potential advantages like decreased blood loss, reduced surgical time, less chances of dura tear. It is a prospective study of 100 patients operated for spinal decompression using either through conventional method or UBS. Patients` demographics profile, duration of surgery, blood loss, complications, Oswestry disability index (ODI), Visual Analogue Scale (VAS) pre operatively and 1,6,12 month post operatively were noted. Out of 100 patients, 46 were operated for laminectomy using conventional methods and 54 were operated using UBS. Average blood loss during surgery using conventional methods was $300 \mathrm{ml}$ and with UBS was $90 \mathrm{ml}$. Average duration of surgery using conventional method was 120 minutes while with UBS was 90 minutes. The difference was statistically significant $(p<0.05)$. Average post-operative VAS score for conventional method was 2.33 and for UBS was 2.12. The difference was not statistically significant $(p>0.05)$. According to ODI score, excellent to good score in conventional method was $64 \%$ and excellent to good score in UBS is $78 \%$. But overall there was no statistically significant difference $(\mathrm{p}>0.05)$. Two patients out of 54, operated using conventional methods had dura tear and none of the patient operated with UBS experienced dura tear. The ultrasonic bone scalpel is a unique surgical device that acts by principle of rapid back and forth movement along with continuous saline irrigation system, thereby reducing heat production and having more selectivity to hard tissue thus causing less damage to the soft tissue, lesser blood loss and short surgical time.

Keywords: Laminectomy, dural tear, ultrasonic bone scalpel

\section{Introduction}

A laminectomy is a surgical procedure that removes a portion of vertebral bone called lamina to decompress the cord or nerve root. Spinal stenosis is the most common indication of laminectomy. Dural tear is an unintended iatragonic complication during spinal surgeries. Management of dural tears requires intraoperative repair with or without fibrin glue or fat graft placement and post-operative flat bed rest with drain placement to reduce cerebrospinal fluid leakage. However, despite this measures complications resulting from dural tears and infection like orthostatic headaches, wound necrosis, infection etc. may develop ${ }^{[1]}$.

There is increased incidence of dural injuries with the conventional methods of spinal decompression like osteotomes and nibblers in patients with congenital stenotic lesion in which there is short pedicle with medially placed facet and fluorotic spine where there is thin dura and harder bone. Use of high-speed drills and diamond burrs may increase the heat production and cause damage to soft tissue. Moreover, the vibration produced by high speed drills (HSDs) may cause fatigue to surgeons and produce discomfort ${ }^{[2,4]}$.

Ultrasound Bone Scalpel (UBS) in spinal surgeries especially for laminectomy is increasing in popularity due to numerous potential advantages like reduced blood loss, reduced time of surgery and very less chances of complications like dura tear and neurologic complications. The purpose of this study is to evaluate and compare results of laminectomy using ultrasound bone scalpel vs. conventional methods. 


\section{Materials and Methods}

It is a prospective study of 100 patients operated for spinal decompression between january-2016 to January-2018, using either conventional method or ultrasound bone scalpel at B.J Medical College, Ahmedabad. Patients demographics profile, disease type, duration of surgery, blood loss (measured by weighting gauze piece and measuring drain output during surgery), complications, functional outcome as measured by Oswestry disability index score (ODI) and Visual Analogue Scale (VAS). Inclusion criteria are 1) Patient between 20-80 years of age 2) Clinically and radiologically proven spinal canal stenosis 3) Patients with 1 year post-operative followup. Exclusion criteria are 1) Patients having infective etiology like Koch`s or metastasis 2) Patient with traumatic canal stenosis 3) Patients with immunocompromised state.

Preoperatively radiological investigations were done in the form of X-rays of respected segments with AP and lateral views And MRI of respective segment with whole spine screening. Routine blood investigations in the form of CBC/RFT/HIV/HBsAg were done. All patient's neurological function on admission was graded according to Frankel et al grading. Functional assessment done according to Oswestry Disability Score (ODI) and Visual Analogue Score (VAS) preoperatively.

All included patients were operated through posterior approach of spine and multilevel laminectomy was done for different indications. Measurement of intraoperative blood loss, duration of surgery, was done in both the group of patients. Immediately post-surgery, routine lateral and anteroposterior radiographs were done to assess the extent of laminectomy, neurological assessment and ODI and VAS scores assessment was done and any complications were noted. All patients were followed up at 1, 6 months and 12 months and at each follow up neurological examination and history of improvement in symptoms, any complications, evaluation of plain radiograph and functional assessment according to ODI and VAS score was noted.

The UBS works on the principle of micro motion of the cutting blade at a frequency of around 22,500 per second. The ultrasonic cutting blade does not bend when it comes in contact with bone but instead transfers the energy to the point of contact with bone and cuts it ${ }^{[3,5]}$. On contact with soft tissue like dura and nerve roots, these soft tissues bend and move away from the tip of cutting blade thereby preventing transfer of high energy to them and avoiding injury.

\section{Results}

In this study, 100 patients were included. 46 were operated for laminectomy using ultrasound bone scalpel (UBS) and 54 patients operated using conventional method like high speed drills. Average follow up duration is 19 months (range from $14-24$ months). Maximum numbers of patients were found in age group of 51-60(42\%) with mean age of 50.85 years. Male to female ratio is almost 1:1. Maximum number of patients operated for laminectomy in our study were having cervical pathology (48\%), followed by lumbar level (38\%).

Average blood loss during surgery using conventional methods was $400 \mathrm{ml}$ and with UBS was $150 \mathrm{ml}$. Average duration of surgery using conventional method is 120 minutes while that of ultrasound bone scalpel was 90 minutes. The difference is statistically significant with $p<0.05$.

Average post-operative VAS score for conventional method is 2.33 and for UBS is 2.12 . The difference is not statistically significant $(p>0.05)$.

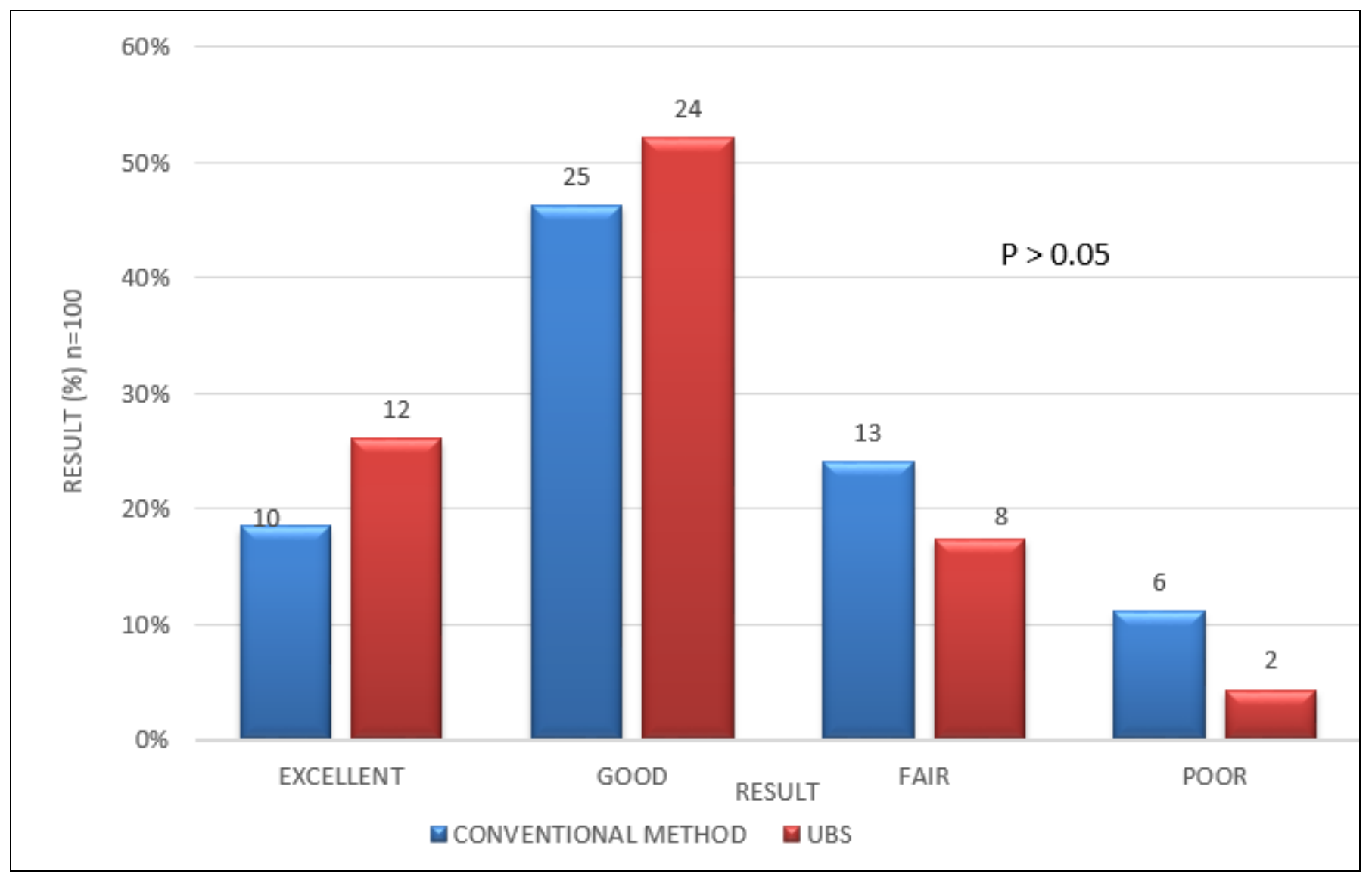

Fig 1: Results of patients treated with conventional method and UBS according to ODI score 
Here, According to ODI score, excellent to good score in conventional method is $64 \%$ and excellent to good score in UBS is $78 \%$. But overall there is no statistically significant difference $(p>0.05)$.

In our study, 2 patients out of 54 patients operated using conventional methods had incidences of dura tear and none of the patient out of 46 patients operated with UBS experienced dura tear. However, none of these patients developed any neurological deficits due to dural tear. There were 2 cases of superficial wound infection in conventional method group and 1 case in UBS group, which were treated with systemic antibiotics and local wound debridement.

\section{Discussion}

In our study, majority of patients were between age group of 51-60 years with mean age of 50.85 years. It's because at this age the disc is more prone to degenerative changes. In younger patients, younger than age of 40 years, resilience of the disc protects it from degeneration. In our study, the canal stenosis was found to be slightly more common in males. Male: female ratio was 1.04. This because, the men are exposed to more physical stresses, especially twisting and lifting without the knowledge of using their muscles of back properly.

According to our study, the mean duration of the surgery was 90 minutes with those operated with UBS as compared to the mean duration of 120 minutes for those operated with conventional methods. This is because surgeon can work freely on spine with less risk of damaging soft tissue. Modi et $a l^{[6]}$ in their study found the average surgical duration to be 90-120 minutes in thoracic, 60-75 minutes in lumbar and 4560 minutes in cervical surgeries. Mehmet et al. ${ }^{[7]}$ in their study found the mean laminectomy time for cervical spondylotic myelopathy was $2.2 \pm 0.4 \mathrm{~min} / \mathrm{level}$ using UBS and the mean laminectomy time was $7.4 \pm 2.6 \mathrm{~min} /$ level using High Speed Drill. The above studies support our findings that UBS reduces the duration of the surgery.

The average blood loss was $150 \mathrm{ml}$ for UBS as compared to $400 \mathrm{ml}$ for conventional method. The reason for less blood loss is that the UBS works with irrigation saline system continuously which acts as haemostasis. Mehmet et al. ${ }^{\text {[7] }}$ found the average blood loss during cervical laminectomy to be $380 \mathrm{ml}$ with HSD as compared to $180 \mathrm{ml}$ with UBS. Modi et al. ${ }^{[6]}$ in their study found average blood loss during cervical laminectomy to be $250 \mathrm{ml}$. The above studies support our findings that UBS reduces the blood loss as compared to HSD.

Complications like dura tear was less frequently noted as compared to conventional method. Bydon et al. ${ }^{[2]}$ described the rate of incidental durotomy to be $5.7 \%$ while performing laminectomy using UBS while Modi et al. ${ }^{[6]}$ described it to be $5 \%$ in his study. In our study, 2 patients out of 54 patients operated using conventional methods had incidences of dura tear and none of the patient out of 46 patients operated with UBS experienced dura tear which indicates the use of UBS is safe and causes less neural damage. There is no statistically significant difference between the two methods in terms of functional outcome as measured by ODI and VAS score.

However, there are certain limitations to our study. The study is not a randomised control trial and it is not a blinded study. The sample size is also small. Based on our study, a multicentre randomised control double blinded trial can provide a more accurate analysis of the advantages of UBS system.

\section{Conclusion}

The ultrasonic bone scalpel is a unique surgical device that acts by principle of rapid back-and -forth movement along with continuous saline irrigation system there by reducing heat production and having more selectivity to hard tissue thus causing less damage to the soft tissue and lesser blood loss. Though surgeon's skill may be an important factor while performing the laminectomy procedure either with UBS or conventional method like Kerrison or high-speed drills, the use of UBS has an advantage of added safety. Our study highlights the advantages of UBS for spinal laminectomy over conventional methods in terms of less blood loss during surgery, shorter duration of surgery, less chances of complications like dura tear and neurological injury.However, currently UBS system is costly and requires additional maintenance thereby limiting its widespread use in routine spine surgeries.

\section{References}

1. Hazer DB, Ya et al. "Technical Aspects on the Use of Ultrasonic Bone Shaver in Spine Surgery: Experience in 307 Patients." BioMed Research International 2016, 8.

2. Bydon M, Xu R et al. "Safety of spinal decompression using an ultrasonic bone curette compared with a highspeed drill: outcomes in 337 patients." Journal of Neurosurgery: Spine. 2013; 18(6):627-633.

3. Nakagawa H, Kim SD et al. "Technical advantages of an ultrasonic bone curette in spinal surgery." Journal of Neurosurgery: Spine. 2005; 2(4):431-435.

4. $\mathrm{Hu} \mathrm{X}$, Ohnmeiss DD et al. "Use of an ultrasonic osteotome device in spine surgery: experience from the first 128 patients." European Spine Journal. 2013; 22(12): 2845-2849.

5. Al-Mahfoudh R, Qattan E et al. "Applications of the ultrasonic bone cutter in spinal surgery-our preliminary experience." British journal of neurosurgery. 2014; 28(1): 56-60.

6. Modi JV, Patel KR, Patel Z, Soman SV, Tankshali KV. Spinal Decompression using Ultrasonic Bone Scalpel: A Novel Ultrasonic Surgical Device. J Spinal Surg. 2016; 3(4):140-143.

7. Onen MR, Yuvruk E et al. "The reliability of the ultrasonic bone scalpel in cervical spondylotic myelopathy: a comparative study of 46 patients." World neurosurgery. 2015; 84(6):1962-1967. 